This item was submitted to Loughborough's Research Repository by the author.

Items in Figshare are protected by copyright, with all rights reserved, unless otherwise indicated.

\title{
Sensitivity analysis of LES-CMC predictions of piloted jet flames
}

PLEASE CITE THE PUBLISHED VERSION

http://dx.doi.org/10.1016/j.ijheatfluidflow.2012.11.007

PUBLISHER

(c) Elsevier

VERSION

AM (Accepted Manuscript)

PUBLISHER STATEMENT

This work is made available according to the conditions of the Creative Commons Attribution-NonCommercialNoDerivatives 4.0 International (CC BY-NC-ND 4.0) licence. Full details of this licence are available at: https://creativecommons.org/licenses/by-nc-nd/4.0/

\section{LICENCE}

CC BY-NC-ND 4.0

\section{REPOSITORY RECORD}

Garmory, Andrew, and E. Mastorakos. 2015. "Sensitivity Analysis of LES-CMC Predictions of Piloted Jet Flames”. figshare. https://hdl.handle.net/2134/17321. 


\title{
Sensitivity Analysis of LES-CMC Predictions of Piloted Jet Flames
}

\author{
A. Garmory ${ }^{\mathrm{a}, *}$, E. Mastorakos ${ }^{\mathrm{a}}$ \\ ${ }^{a}$ Hopkinson Laboratory, Department of Engineering, University of Cambridge, \\ Cambridge CB2 1PZ, United Kingdom
}

\begin{abstract}
The sensitivity of Large Eddy Simulation with Conditional Moment Closure (LES-CMC) simulations of the Sandia piloted jet Flames D and F to various parameters have been investigated. It was found that while an LES grid may sufficiently resolve velocity fields, the conditional scalar dissipation rate obtained may still be affected by grid size due to the calculation of sub-grid scalar dissipation rate, and this can affect the degree of localised extinction predicted. A study of the relative size of the terms in the CMC equation during an extinction/reignition event showed that transport, including in the cross stream direction, plays a key role. The results are sensitive to the choice of inlet boundary conditions as extinction is only observed when the inert-mixing distributions in mixture fraction space are used as inlet conditions for the CMC equation in the primary jet and air jets.
\end{abstract}

Keywords:

LES, CMC, extinction, re-ignition, non-premixed

\footnotetext{
*Corresponding author. Present address Department of Aeronautical and Automotive Engineering, Loughborough University, Loughborough, LE11 3TU, United Kingdom Email address: A.Garmory@lboro.ac.uk (A. Garmory )
} 


\section{Introduction}

Due to its superior ability to predict the details of turbulent mixing, Large Eddy Simulation is increasingly being used to study turbulent combustion in a variety of industrial applications. Even with the greater spatial resolution of LES compared to RANS the combustion process still takes place on a scale which cannot be resolved by the grid, and as such some form of turbulent combustion modelling must be employed. These include steady (Di Mare et al., 2004) and unsteady (Pitsch and Steiner, 2000) flamelet models, the flamelet/progress variable (FPV) model (Pierce and Moin, 2004) and the Stochastic Fields or Eulerian Monte Carlo method (Mustata et al., 2006). The Conditional Moment Closure model, discussed later, is another advanced model that is being used for flames with strong turbulence-chemistry interactions.

In order for any such model to become a useful engineering tool it is important that they are validated against detailed measurements and that the models' sensitivity to modelling choices and parameters are investigated. The Sandia piloted jet flames (Barlow and Frank, 1998) provide detailed experimental data for both scalar and velocity fields and consequently have been widely used for this sort of validation work. Data is available for conditions ranging from a flame with very little local extinction (Flame D) to one that is close to global extinction (Flame F). RANS - Muliple Mapping Conditioning (MMC) simulations of Flame D have been performed by Vogiatzaki et al. (2011) in order to determine the value of modelling parameters which give the best agreement with conditional variance of temperature and various species mass fractions. Previous studies using transported PDF methods in RANS 
have produced good agreement with experiment (Lindstedt et al., 2000; Xu and Pope, 2000) for Flame F and also revealed the sensitivity of this Flame $\mathrm{F}$ to the chosen chemical mechanism (Cao and Pope, 2005). These studies were useful in determining the parameters needed in RANS-PDF modelling to give accurate results, a process that is now being undertaken for LES studies. The presence of localised extinction in Flame E has successfully been predicted in Ihme and Pitsch (2008) using the FPV model. The Eulerian stochastic fields PDF method has been used in Jones and Prasad (2010) to successfully predict the presence of localised extinctions in Flame F

Conditional Moment Closure (CMC), which is the subject of this paper, has previously been used in an LES context for Sandia Flame D (NavarroMartinez et al., 2005), bluff-body steady flames (Navarro-Martinez and Kronenburg, 2007), autoigniting jets (Navarro-Martinez and Kronenburg, 2009) and for spark ignition problems (Triantafyllidis et al., 2009). An LES-CMC formulation solving the $\mathrm{CMC}$ equations on a $3 \mathrm{D}$ grid (i.e. resolving variations of conditional average in three dimensions rather than using cross stream averaging) has been used to successfully predict the presence of localised extinction and reignition events in both Sandia Flame F (Garmory and Mastorakos, 2011) and the Delft III piloted jet flame (Ayache and Mastorakos, 2012). It was shown that the model can successfully predict the occurence of localised extinction, and the resulting statistics of species mass fractions and temperature.

The purpose of this paper is to revisit the simulations of Sandia Flames $\mathrm{D}$ and $\mathrm{F}$ in order to investigate the sensitivity of the results to the modelling choices used. This will build confidence to the use of the LES-CMC approach 
for more complex flames of practical significance. An extended discussion of how the CMC method predicts extinction/reignition and how this may influence its accuracy is also presented. In the next section the formulation of the LES-CMC method is briefly covered and its numerical implementation is discussed. Particular emphasis is placed on modelling choices where more than one option is employed here. This is followed by results obtained using these choices with a discussion of them. The conclusions of this work are summarised in the last section of the paper.

\section{Formulation}

\subsection{LES with $C M C$}

In the CMC method the assumption is made that while reactive scalar values might fluctuate strongly, their fluctuations about a value conditionally averaged on the value of some conserved scalar will be small. Hence the fluctuations of all reactive scalars can be related to that of a conserved scalar, usually mixture fraction in non-premixed combustion. Transport equations for the conditional averages are solved on a grid considerably coarser than that used for the LES. The required scalars for the LES code are then found by using their conditional average and a local mixture fraction PDF. In this paper we examine the ability of LES-CMC to predict localised transient extinction and re-ignition events within a turbulent non-premixed flame.

The LES-CMC code developed in Triantafyllidis et al. (2009); Triantafyllidis and Mastorakos (2010) and used in Garmory and Mastorakos (2011); Ayache and Mastorakos (2012) has been employed here. Filtering the governing equations for the flow yields equations for filtered mass 


$$
\frac{\partial \bar{\rho}}{\partial t}+\frac{\partial\left(\bar{\rho} \widetilde{u}_{i}\right)}{\partial x_{i}}=0
$$

momentum

$$
\frac{\partial\left(\bar{\rho} \widetilde{u}_{i}\right)}{\partial t}+\frac{\left(\bar{\rho} \widetilde{u}_{i} \widetilde{u}_{j}\right)}{\partial x_{i}}=-\frac{\partial \bar{p}}{\partial x_{j}}+\frac{\partial \widetilde{\tau}_{i j}}{\partial x_{i}}-\frac{\partial\left(\bar{\rho} \tau_{i j}^{r}\right)}{\partial x_{i}}
$$

and a conserved scalar, mixture fraction, $\xi$,

$$
\frac{\partial(\bar{\rho} \widetilde{\xi})}{\partial t}+\frac{\left(\bar{\rho} \widetilde{\xi} \widetilde{u}_{i}\right)}{\partial x_{i}}=\frac{\partial}{\partial x_{i}}\left(\bar{\rho}\left(D+D_{t}\right) \frac{\partial \widetilde{\xi}}{\partial x_{i}}\right)
$$

The term $\tau_{i j}^{r}$ in Eq. (1) is the sub-grid scale stress tensor and is modelled by the dynamic Smagorinsky (Germano et al., 1991). In Eq. (3) a gradient model has been used to model the sub-grid scale flux $\widetilde{u_{i} \xi}-\widetilde{u}_{i} \widetilde{\xi}=-D_{t} \partial \widetilde{\xi} / \partial x_{i}$. $D_{t}=\nu_{t} / \mathrm{Sc}_{\mathrm{t}}$ is the turbulent diffusivity, and $\mathrm{Sc}_{\mathrm{t}}=0.7$ is the turbulent Schmidt number, assumed here to be constant (Branley and Jones, 2001). It is also necessary to obtain the sub-grid scale variance of the mixture fraction. Here this has been done by assuming a gradient type model:

$$
\widetilde{\xi^{\prime \prime 2}}=C_{V} \Delta^{2} \frac{\partial \widetilde{\xi}}{\partial x_{i}} \frac{\partial \widetilde{\xi}}{\partial x_{i}}
$$

$C_{V}$ is a constant whose value is determined dynamically according to Cook and Riley (1994); Pierce and Moin (1998). $\Delta$ represents the grid spacing or filter width.

When the CMC model (Klimenko and Bilger, 1999) is used, equations are solved for the conditionally filtered reactive scalars, in a non-premixed case it is natural that the conditioning be done on $\xi$. The filtered value of the variable $f$ can then be obtained by integration over $\eta$-space (Triantafyllidis 
et al., 2009):

$$
\widetilde{f}=\int_{0}^{1} \widetilde{f \mid \eta} \widetilde{\mathcal{P}}(\eta) d \eta
$$

We assume here that $\widetilde{\mathcal{P}}(\eta)$ has a $\beta$-function shape, which can be calculated based on the $\widetilde{\xi}$ and $\widetilde{\xi^{\prime \prime}}$.

The CMC equations can be derived by filtering the transport equations for the reactive scalars $Y_{\alpha}$ (Navarro-Martinez et al., 2005). Using the primary closure assumption, the CMC equation becomes

$$
\frac{\partial Q_{\alpha}}{\partial t}+\widetilde{u_{i} \mid \eta} \frac{\partial Q_{\alpha}}{\partial x_{i}}=\widetilde{N \mid \eta} \frac{\partial^{2} Q_{\alpha}}{\partial \eta^{2}}+\widetilde{\omega_{\alpha} \mid \eta}+e_{f}
$$

where $Q_{\alpha}=\widetilde{Y_{\alpha} \mid \eta}$ is the conditionally filtered reactive scalar, $\widetilde{u_{i} \mid \eta}$ is the conditionally filtered velocity, $\widetilde{N \mid \eta}$ is the conditionally filtered scalar dissipation rate, $\widetilde{\omega_{\alpha} \mid \eta}$ is the conditionally filtered reaction rate, while the term

$$
e_{f}=-\frac{1}{\bar{\rho} \widetilde{\mathcal{P}}(\eta)} \frac{\partial}{\partial x_{i}}\left[\bar{\rho} \widetilde{\mathcal{P}}(\eta)\left(\widetilde{u_{i} Y_{\alpha} \mid \eta}-\widetilde{u_{i} \mid \eta} Q_{\alpha}\right)\right]
$$

is the sub-grid scale conditional flux and accounts for the conditional transport in physical space.

In this work the conditional scalars are the mass fractions of chemical species plus absolute enthalpy. This means a conditional temperature equation does not need to be solved as it can be determined from absolute enthalpy and species composition. All the species are assumed to have equal diffusivities and the Lewis number is assumed to be equal to one. A gradient model is used for the sub-grid scale conditional flux $\widetilde{u_{i} Y_{\alpha} \mid \eta}-\widetilde{u_{i} \mid \eta} Q_{\alpha}=-D_{t} \frac{\partial Q_{\alpha}}{\partial x_{i}}$ (Navarro-Martinez et al., 2005). This model has given reasonable results in problems with significant spatial gradients of the conditional averages in LES 
of ignition (Triantafyllidis et al., 2009). Equation 6 without spatial transport terms and with a prescribed $\widetilde{N \mid \eta}$ has also been solved to give reference "laminar flamelet" solutions, denoted as "0D-CMC", and for initialisation.

The terms $\widetilde{u_{i} \mid \eta}$ and $\widetilde{N \mid \eta}$ are unclosed and require modelling. The simple assumption that the conditional velocity is equal to the unconditional is made here, $\widetilde{u_{i} \mid \eta}=\widetilde{u_{i}}$. The accuracy of this assumption is clearly open to question, however there appears to be no obvious alternative at the present to use with LES-CMC. The simple linear model used in RANS-CMC (Klimenko and Bilger, 1999) does not seem appropriate for the more complex mixing field in LES. Investigation of the effect of conditional velocity model on LESCMC is clearly needed, but it is beyond the scope of this present work.

The Amplitude Mapping Closure (AMC) model is used to model the conditional scalar dissipation rate (O'Brien and Jiang, 1991). These modelling choices have previously been applied with some success to spark ignition simulations in Triantafyllidis et al. (2009). According to the AMC model, the conditional scalar dissipation rate has a given shape in mixture fraction space, which is scaled according to the local value of the unconditional scalar dissipation rate. Hence, $\widetilde{N \mid \eta}=N_{0} G(\eta)$, where $G(\eta)=\exp \left(-2\left[\operatorname{erf}^{-1}(2 \eta-1)\right]^{2}\right)$ and $N_{0}=\widetilde{N} / \int_{0}^{1} \widetilde{\mathcal{P}}(\eta) G(\eta) d \eta$, where $\widetilde{N \mid \eta}$ is the conditional and $\widetilde{N}$ is the filtered scalar dissipation rate. The total scalar dissipation rate $\widetilde{N}$ is found by summing the resolved and sub-grid contributions where the resolved component is

$$
\widetilde{N}_{\text {res }}=D\left(\frac{\partial \widetilde{\xi}}{\partial x_{i}}\right)^{2}
$$

and the sub-grid component is found by assuming that a mixing timescale 
is proportional to a velocity timescale (Jiménez et al., 2001) for the sub-grid $\tau_{t}=\Delta^{2} / \nu_{t}$ :

$$
\widetilde{N}_{s g s}=\frac{1}{2} C_{N} \frac{\nu_{t}}{\Delta^{2}} \widetilde{\xi^{\prime \prime 2}}
$$

The constant $C_{N}$ was adjusted in Garmory and Mastorakos (2011) to give a good match with the conditional scalar dissipation rate experimental data available for Flame D. The value of $C_{N}$ found was 42 and this is used throughout this paper.

Equation (6) is solved on a coarser grid than the LES and hence the information for $\widetilde{u_{i} \mid \eta}$ and $\widetilde{N \mid \eta}$ needed in the CMC must be averaged from the LES. This is done (Triantafyllidis and Mastorakos, 2010) by associating each LES cell with its closest CMC grid point, the required data for each CMC node is then mass weighted averaged over all LES cells associated with that node. In turn each LES cell uses the conditional averages, $Q_{\alpha}$, from its associated CMC node to find its new value of temperature and density. The conditional scalar dissipation rate is found by calculating the mass averaged $\widetilde{N}$ and $\mathcal{P}(\eta)$ for the whole CMC cell and applying the AMC model at the CMC level. The effect of using this method, rather than applying the AMC model at the LES level and averaging this, was investigated by averaging experimental data at different spatial resolutions in Triantafyllidis and Mastorakos (2010). They found that the method used here gave results that were comparable to and in some cases better than the other method. This method of applying models for the scalar dissipation rate and the condition velocity at the CMC level, rather than the LES level and then PDF averaging, also avoid the numerical problem of undefined conditional values when the PDF tends to zero. 


\subsection{Chemical Source Term}

First order closure is provided for the chemical reaction rate: $\widetilde{\omega_{\alpha} \mid \eta}=$ $\omega_{\alpha}\left(Q_{1}, Q_{2}, \ldots, Q_{n}\right)$, where $n$ is the number of reacting scalars. The ARM2 chemistry has been used. This mechanism is a systematically reduced version of the GRI mechanism where 19 reactive species plus temperature and 15 steps (Sung et al., 1998). This mechanism has previously been found to give good accuracy without a prohibitive computational cost in Cao and Pope (2005).

\subsection{Numerical Methods and Simulation Details}

The LES equations are solved by the in-house Rolls-Royce code PRECISE, which is a finite-volume, low-Mach number code with block-structured mesh (James et al., 2006). The CMC model has been implemented in PRECISE with coupling achieved through density and temperature (Triantafyllidis et al., 2009). The CMC code is a finite difference code. A three-stage operator splitting scheme is applied whereby spatial transport is solved first, followed by transport in $\eta$-space and finally chemistry. The solver VODPK (Brown and Hindmarsh, 1989) has been used to integrate the resulting system of ODE's. Parallelisation is achieved in PRECISE through domain decomposition and in the CMC code by dividing nodes in $\eta$-space between processors for the transport step and dividing spatial nodes between processors for the final two steps.

The Sandia piloted jet diffusion Flames $\mathrm{D}\left(U_{j}=50 \mathrm{~m} / \mathrm{s}\right)$ and $\mathrm{F}\left(U_{j}=\right.$ 99m/s) (Barlow and Frank, 1998) have been modelled. Flame D has minimal local extinction whereas $\mathrm{F}$ has a large amount of local extinction and is close to global extinction. The jet has a diameter of $7.2 \mathrm{~mm}$ and contains 
$25 \%$ methane $-75 \%$ air by volume. This is surrounded by a pilot of outer diameter $18.2 \mathrm{~mm}$. The LES boundary conditions are experimental velocity profiles across the jet and pilot from Schneider et al. (2003). No fluctuations are added to the inlet velocity but previous studies (Navarro-Martinez et al., 2005) have not found this to have a large effect as most of the turbulence in the jet is created in the shear layer. The mixture fraction is set to 1.0 in the jet, 0.0 in the co-flow and 0.27 in the pilot. This value is the mixture fraction that gives the pilot temperature $(1880 \mathrm{~K})$ and composition in a calculation of a low scalar dissipation rate laminar diffusion flame.

In order to test the sensitivity of the results two LES grids have been used. The first, 'coarse' grid covers a domain extending 80D in the axial and $20 \mathrm{D}$ in the radial directions. The grid is an 'O-ring' mesh using a minimum grid spacing of $0.25 \mathrm{~mm}$ across the pilot with spacing expanding smoothly downstream so that a total of approximately 1.3M nodes are used. The second 'fine' grid covers 40D in the axial and 20D in the radial directions. The same number of nodes are used in the axial direction, doubling the axial resolution, and the radial and azimuthal resolution is increased, giving a total of $2 \mathrm{M}$ nodes.

With the LES-CMC method, as employed here, two computational meshes are required; a fine mesh for the LES and a much coarser mesh for the CMC. The rationale behind the coarse CMC mesh is that conditional means will in general vary over much longer lengthscales than unconditional values. Two CMC spatial grids have been used to test the sensitivity to this factor. Both grids use 51 nodes in $\eta$-space, it was found that the results for 0D CMC calculations (i.e. only chemistry and scalar dissipation rate) where virtually 
unchanged if 101 nodes were used, so we believe that the results are independent of $\eta$-space resolution. The first grid, 'CMC grid 1,' $23 \times 23 \times 23$ nodes in an orthogonal mesh in physical space. The grid in the axial direction has an initial spacing of $3 \mathrm{~mm}$ until 3 jet diameters and then expands with a successive ratio of 1.3. The comparatively high number of nodes in the cross stream directions allow us to resolve the pilot and jet assembly with $9 \times 9$ of the nodes covering this region. The fine LES grid uses the same CMC grid spacing up to 40D, hence less CMC nodes are needed for this case. A second CMC grid, 'CMC grid 2,' uses the same spacing in the cross stream directions but uses 25 nodes in the axial direction with initial spacing of $7.2 \mathrm{~mm}$ $(y / D=1)$ up to $y / D=15$ and an expansion after this point. Tables $1 \& 2$ summarises these details.

\begin{tabular}{|l|l|c|}
\hline LES Grid & $\begin{array}{l}\text { Overall Dimension } \\
\text { (Radial X Axial) }\end{array}$ & Total Cells \\
\hline Coarse & $20 \mathrm{Dx} 80 \mathrm{D}$ & $1.3 \mathrm{M}$ \\
Fine & $20 \mathrm{Dx} 40 \mathrm{D}$ & $2 \mathrm{M}$ \\
\hline
\end{tabular}

Table 1: Summary of LES grids.

\begin{tabular}{|l|c|l|}
\hline CMC Grid & Cross jet cells & Axial Spacing \\
\hline CMC 1 & $23 \times 23$ & $\begin{array}{l}3 \mathrm{~mm} \text { until } 3 \text { jet diameter } \\
\text { then successive ratio of } 1.3 . \\
\text { CMC 2 }\end{array}$ \\
$\begin{array}{l}\text { Constant spacing of } 7.2 \mathrm{~mm} . \\
(y / D=1) \text { up to } y / D=15\end{array}$ \\
\hline
\end{tabular}

Table 2: Summary of CMC grids. 
As well as the spacing of the CMC grid, the numerical method used to evaluate the transport terms is investigated. Diffusion in physical and $\eta$ space is discretised using a second-order accurate central difference scheme, while convection uses either an Upwind Differencing Scheme (UDS) or a TVD scheme (Chung, 2002).

As well as a separate grid, the CMC equations need to have separate boundary conditions. At the inlet a boundary value must be chosen for each species at every value of mixture fraction. Another way to think of this is that inlet 'flamelets' need to be specified on the inflow boundaries. In the case studied here, at the inlet only a single value of mixture fraction is present at any point and hence the inlet conditional averages for the rest of mixture fraction space are not fully defined. As such the selection of CMC inlet boundary conditions represents a modelling choice. Two choices are investigated here; firstly inert flamelets (291K at all $\xi$ values) in the jet and co-flow and a burning flamelet that gives the correct temperature at $\xi=0.27$ in the pilot. Secondly, the burning flamelet is used for all CMC inlet nodes. With the latter, the delta function mixture fraction PDF's at 0.0, 0.27 and 1.0 will still lead to the correct unconditional boundary values.

A final sensitivity test was to apply an increase of $20 \%$ to the inlet velocity profile. The choices for each simulation are shown in Table 3. Note that simulations D1 and F1 are those presented in Garmory and Mastorakos (2011). The timestep used was $\Delta t=2 \times 10^{-6} \mathrm{~S}$. The simulations were carried out on $323.0 \mathrm{GHz}$ dual core processors each with $2 \mathrm{~GB}$ of RAM per core. $7.2 \mathrm{~ms}$ of simulated time could be produced in 24 hours for the coarse LES grid with CMC grid 1. 


\begin{tabular}{|l|rr|rrrrr|}
\hline Case & D1 & D2 & F1 & F2 & F3 & F4 & F5 \\
\hline Flame & $\mathrm{D}$ & $\mathrm{D}$ & $\mathrm{F}$ & $\mathrm{F}$ & $\mathrm{F}$ & $\mathrm{F}$ & $\mathrm{F}$ \\
LES grid & $\mathrm{C}$ & $\mathrm{F}$ & $\mathrm{C}$ & $\mathrm{C}$ & $\mathrm{C}$ & $\mathrm{C}$ & $\mathrm{C}$ \\
Convection & TVD & TVD & TVD & UDS & TVD & UDS & UDS \\
CMC grid & 1 & 1 & 1 & 1 & 2 & 1 & 1 \\
CMC boundary & $\mathrm{S}$ & $\mathrm{S}$ & $\mathrm{S}$ & $\mathrm{S}$ & $\mathrm{S}$ & $\mathrm{O}$ & $\mathrm{S}$ \\
Velocity $(\mathrm{m} / \mathrm{s})$ & 99 & 99 & 99 & 99 & 99 & 99 & 119 \\
\hline
\end{tabular}

Table 3: Modelling choices for simulations. For CMC boundary, 'standard' refers to burning flamelets only in the pilot and inert in jet and co-flow, 'Option' refers to burning flamelets at all inlet nodes.

\section{Results and Discussion}

3.1. Flame D

Experimental investigation of Flame D shows that there is very little local extinction in this flame (Barlow and Frank, 1998). This makes its suitable for testing the ability of the code to predict the correct flow field and flame temperature. The velocity field predicted by cases D1 and D2 (which has a finer LES grid) compared to experimental data taken from Schneider et al. (2003) is shown in Fig. 1. The data from the LES is found by averaging data taken every $0.2 \mathrm{~ms}$ of simulated time for a total of $20 \mathrm{~ms}$ for D1 and $15 \mathrm{~ms}$ for the shorter domain of D2. Data is also averaged in the azimuthal direction to give the radial profiles shown in Fig. 1. Overall we see that reasonable agreement is observed between the predicted LES velocity field and experimental data. Both grids predict overpredict the rate at which the pilot is mixed out into the co-flow and the velocity RMS is 
underpredicted early in the jet. The finer grid, as expected, shows better agreement, particularly along the centre line, however the agreement for D1 seems overall to be acceptable.

One of the key modelling issues with $\mathrm{CMC}$ is the conditional scalar dissipation rate. Experimental data for conditional scalar dissipation rate (Karpetis and Barlow, 2005) is available for comparison with simulations. To find a representative conditional average for a particular axial location from the CMC data, all the conditional averages at each CMC node at that axial position were averaged using PDF-weighted averaging, such that:

$$
\widetilde{\phi \mid \eta}^{*}=\frac{\int \widetilde{\phi \mid \eta} \widetilde{\mathcal{P}(\eta) \rho \mid \eta} d V}{\int \widetilde{\mathcal{P}(\eta)} \widetilde{\rho \mid \eta} d V}
$$

where the integration is over all $\mathrm{CMC}$ nodes in all the points in time that are to be averaged. Results for $\widetilde{N \mid \eta}$ in Flame D are shown in Fig. 2. The CMC data is taken from the nearest axial node position to the point of interest, i.e. the CMC nodes that the LES cells at that point use. We can see that case $\mathrm{D} 1$ which uses a coarser CMC grid overpredicts $\widetilde{N \mid \eta}$ at $y / D=7.5$ but underpredicts it at $y / D=15$. It should be noted that adjustment of $C_{N}$ can only increase or decrease $\widetilde{N \mid \eta}$ at all points and hence better agreement cannot be obtained for this grid by this method. The finer grid in D2 gives improved results probably through decreasing the effect of sub-grid terms which have to be modelled. With this grid we see that $C_{N}=42$ is still a reasonable value. It is possible that better universal agreement could be obtained by solving transport equation for the sub-grid variance rather than Eq. (4).

The conditional temperatures predicted by the CMC using both D1 and 
D2 are shown in Fig. 3, again a representative conditional average found using Eq. (10) is shown for each axial location. This is consistent with the experimental data from Barlow and Frank (1998) where conditional averaging has been performed on data taken across the jet. The agreement with experiment is good, with the overprediction of temperature most likely being due to the neglect of radiation effects in the modelling used here (Pitsch and Steiner, 2000). Both D1 and D2 give very similar results here as, even though there are differences in conditional scalar dissipation rate, the flamelets are well away from extinction and hence the dissipation rate does not cause large differences in conditional temperature.

The unconditional temperature from simulations D1 and D2 along the centre line of the jet is shown in Fig. 4 along with the mixture fraction.This has again be found by averaging data taken every $0.2 \mathrm{~ms}$ of simulated time for a total of $20 \mathrm{~ms}$. The excellent prediction of both mean and rms of mixture fraction predicted by the LES for both grids here, coupled with the close agreement for conditional temperature seen in Fig. 2, ensures that there is very good agreement seen for mean and rms of unconditional temperature. Good agreement is also seen for radial profiles of temperature, although they have been omitted here to save space.

\subsection{Flame $F$}

\subsubsection{Statistical Quantities}

LES-CMC was then applied to Flame F, which has a large amount of localised extinction. Case F1 uses exactly the same configuration as D1 with only the inlet boundary conditions (i.e. higher jet velocity) for the LES changed. The results of this were presented in Garmory and Mastorakos 
(2011), where it was seen that the LES-CMC method was capable of predicting local extinction and reignition. For both conditional and unconditional results the correct degree of extinction was seen at $y / D=7.5$ (as shown by a decrease in the time averaged temperatures) but by $y / D=15$ the level of extinction predicted was not enough. Interestingly this echoes the Eulerian stochastic fields results seen in Jones and Prasad (2010) (the only other LES of Sandia F in the open literature the authors are aware of), where the averaged level of extinction was also underpredicted at $y / D=15$.

The sensitivity of the simulations of Flame $\mathrm{F}$ to various modelling choices has been explored and are shown next, which can help assess the predictive capability of LES-CMC as an engineering tool for flames very close to extinction. These options are described in Section 2.3 and summarised in Table 3. We also present simulations for a hypothetical flame of jet velocity $20 \%$ higher than that of Flame $\mathrm{F}$ to examine any trends revealed.

The effect of these options is shown in Figs. $5 \& 6$, which show averaged conditional means of temperature and $O H$, found using Eq. (10), at axial locations of $y / D=3,7.5,15$ and 30. Simulation F1 shows very good agreement for conditional temperature, including predicting the reduced temperature due to extinction at $y / d=7.5$, everywhere except $y / d=15$. The conditional $O H$ level is overpredicted with respect to the experiment. The good agreement for temperature when $\mathrm{OH}$ is overpredicted may be an issue with the chemical mechanism used.

The trends with the sensitivity options are the same for both scalars. Increasing the jet velocity in the calculation (F5) leads to an increase in the level of extinction in the flame, seen in the reduced average conditional 
temperature and $O H$ levels at $y / d=3$ and 7.5, which is what would be expected, and demonstrates that the LES-CMC method captures correctly the trend of increasing extinction as the velocity increases. However even with this increased velocity not enough extinction is seen at $y / d=15$ where the conditional temperature is only correctly predicted (for all cases except F5) at high mixture fraction. This may be the reason why complete detachment from the pilot, which occurs in the experiment at a velocity just above 100 $\mathrm{m} / \mathrm{s}$, is not captured by the simulation.

Both changing the discretisation scheme used for convection and changing the CMC grid leads to a change in the level of extinction. We see that the upwind scheme (F2), which is more dissipative than the TVD scheme (F1), leads to a decrease in the level of extinction particularly at 7.5 diameters where the peak conditional temperature can be seen to be too high with this option. As the CMC convection step is computationally inexpensive compared to the chemistry step, using the more accurate TVD scheme does not make the calculation appreciably longer than using the UDS scheme. The importance of the numerical scheme is also shown by the change in results brought about by using an alternative CMC grid, the peak conditional temperature at $y / d=7.5$ is seen to increase by around $150 \mathrm{~K}$ for F3. This reflects the fact that extinctions and reignitions (the latter determine, to a large extent, the degree of extinction seen downstream) depend on the physical transport part of the CMC equation, as discussed in greater detail in 3.2.2.

Figures $5 \& 6$ also show the difference between cases F2 and F4, which only differ in their treatment of CMC inlet boundary conditions. F2 uses 
burning flamelets only for the pilot and inert flamelets in the co-flow and jet, whereas F4 uses burning flamelets at inlet nodes. Case F4 shows virtually no extinction at any axial location. At 7.5 jet diameters the conditional temperatures seen for F4 are those of a fully burning flamelet, while F2 is significantly below this. As early as three jet diameters we see that conditional temperature is overpredicted at high mixture fractions and that this overprediction is seen to continue as far downstream as 30 jet diameters compared to the other cases. This overprediction of temperature at high mixture fraction will lead to underpredictions of density on the jet centre line around 15 diameters which will in turn effect the velocity field.

The effect of the varying degrees of extinction given by the different options on unconditional temperature can be observed in Fig. 7, which shows radial profiles of mean unconditional temperature from all 5 cases at $y / D=7.5$ jet diameters. The LES data has been found by averaging the unconditional temperature from all LES cells at the given radial and axial position taken every $0.2 \mathrm{~ms}$ of simulated time for a total of $20 \mathrm{~ms}$. The peak mean unconditional temperature is lower than the peak conditional temperature due to the varying mixture fraction field. The profiles for cases F1, F2 and F3 are close to each other and to the experimental data at this point. Although the reduced extinction when using UDS rather than TVD can be seen here. Case F5, in which the velocity has been increased, shows a decrease in temperature. While F4, which uses constant CMC inlet boundary conditions, shows an increased temperature, which is consistent with the change in extinction seen in Fig. 5. The peak temperature with F4 is over $300 \mathrm{~K}$ higher than experimental data. We also see that the position of the 
peak temperature moves in cases F4 and F5. This is due to the degree of extinction seen in the simulations being a function of radial position as well as axial position, in agreement with experimental data (see Fig. 9, Garmory and Mastorakos (2011)).

\subsubsection{Discussion of Extinction in $C M C$}

The main mechanism of how localised extinction is captured by the CMC method was briefly presented in Garmory and Mastorakos (2011). In particular it was seen that at a single CMC cell both burning and extinguished flamelets can be found at different times, indicating that transient extinction and reignition events must take place. In this section we will consider how these events are brought about. Figure 8 shows time series of conditional temperature at stoichiometric mixture fraction for several adjacent CMC cells. The central CMC cell of this group, 'A,' is located at $y / D=6.6$ and $r / D=0.9$ and we can see two clear extinction and reignition events during this period. Figure 9 shows the location of this cell on an instantaneous isosurface of stoichiometric mixture fraction coloured by resolved $O H$ mass fraction at a time $(21.8 \mathrm{~ms})$ during one of these extinctions. We can see the extinction of CMC cell 'A' leads to a region where very low levels of $O H$ are seen even where the stoichiometric mixture fraction is found. Also marked on Fig. 9 are the locations of the adjacent cells (B - G) whose time series are also shown in Fig. 8.

In our previous paper (Garmory and Mastorakos, 2011) we showed that extinctions in the CMC grid did not correspond only to high conditional scalar dissipation rates and that spatial transport effects must also play a part. Here we take this a step further by looking at the relative size of 
the different terms in Eq. (6) at different points in time as CMC node 'A' in Fig. 8 undergoes an extinction followed b a reignition. As a constant enthalpy formulation is used to solve the CMC equations, rates of change of temperature are not available, so the rate of formation of $\mathrm{H}_{2} \mathrm{O}$ is used as an indicator of the overall reaction which follows very closely the rate of change of temperature. These are shown in Fig. 10 with (a) just prior to an extinction at $21.28 \mathrm{~ms}$, (b) while the flame is being extinguished at $21.4 \mathrm{~ms}$, (c) during the extinction at $21.8 \mathrm{~ms}$ and (d) as the flamelet is reigniting at $22 \mathrm{~ms}$.

In Fig. 10(a) the production of water by chemical reaction is high and this is approximately balanced by conditional scalar dissipation rate, as would be the case for a steady flamelet. However at this instant the convection term takes a significant negative value which causes the total production rate of water to become negative at this time leading to the extinction observed in Fig. 8. In (b) the temperature has dropped such that the chemical reaction rate has now decreased dramatically and the dominant term is the scalar dissipation rate which causes the temperature of the flamelet to decrease rapidly at this point. For (c) the scalar dissipation rate is balanced by chemistry, convection and spatial diffusion leading to an approximately steady temperature higher than that seen at node ' $\mathrm{F}$,' which is closer to the axis and is always extinguished. The final figure in this series is (d) in which we see the terms have changed such that the overall rate is positive which allows temperature to increase until the flame is reignited. In this last figure we can see that it is important to correctly consider all terms as there is a fine balance between them. 
The effect of convection on causing extinction and reignition has been shown in Fig. 10. If we consider the extinction which occurs at around $21.25 \mathrm{~ms}$ we can see that the only neighbouring cell with a significantly lower conditional temperature (and hence $\mathrm{H}_{2} \mathrm{O}$ mass fraction) is cell ' $\mathrm{F}$ ' which is in the direction of the jet axis. Figure 11 shows the time series of radial velocity used in the CMC for cell 'A.' There is a spike in velocity (i.e. from ' $\mathrm{F}$ ' to 'A') at approximately $21.25 \mathrm{~ms}$ which indicates that the extinction seen here is due to a sudden change in velocity advecting the non-burning flamelet within the jet radially outwards.

In this section we have been able to show that convection, including that in the radial direction, plays a key role in the prediction of localised extinction in Flame F. The importance of transport in predicting extinction/reignition events means that the numerical treatment of convection in the CMC formulation will play an important part in the accuracy of the results it produces. As we have already seen in this paper, changing the discretisation scheme from upwind to TVD and changing the CMC grid have altered the prediction of conditional and unconditional temperature in this flame. Due to the constraints of the structured orthagonal CMC grid and the computing resources available the $\mathrm{CMC}$ grid could not be refined by a large amount here but it may be that with more refinement the extinction would be seen to convect much further downstream.

The importance of cross stream convection in predicting extinction also explains the sensitivity of the results here to the CMC boundary conditions chosen for the primary fuel jet. If an inert (non burning) flamelet is injected in the primary jet then a supply of inert flamelets is available in the centre of 
the jet which a fluctuation in velocity can then cause to be convected radially outward where, in conjunction with high scalar dissipation rate, it can lead to extinction. If burning flamelets are injected across all inlet nodes then this route is no longer available to cause extinction.

\section{Conclusions}

The sensitivity of the LES-CMC method to various factors has been investigated in the context of simulating the Sandia piloted jet Flames D and F. It was found that for Flame D the LES results for velocity fields were very similar for both the fine and coarse LES grids employed here. This shows that the results seen in this work for these quantities can be regarded as grid insensitive. However, it was seen that the conditional scalar dissipation rate extracted from the LES for use in the CMC code was sensitive to the LES mesh. It is likely that using a finer grid improves results for this quantity as it reduces the influence of the modelled sub-grid terms. This suggests that more work is needed on the modelling of unresolved scalar dissipation rate in order to avoid the need to use very fine LES meshes to capture conditional scalar dissipation rate.

By studying the relative size of individual terms in the CMC equation we have been able to show that transport, including in the cross stream direction, plays a key role in the prediction of localised extinction in Flame F. This means that the numerical treatment of transport in the CMC code is of great importance. Changing the CMC grid and the differencing scheme has been seen to have some effect on the results here. But in order to transport extinctions downstream to to 15 jet diameters and beyond, where they are 
seen in Flame F, it may be necessary to have much finer CMC grid spacing in the axial direction. With the orthogonal structured CMC formulation here it would be very difficult to achieve this without reducing cross stream resolution or making the computational load very high. An unstructured CMC grid may be advantageous in this situation.

The biggest sensitivity of these results was seen to be to the choice of CMC boundary conditions. The results using burning flamelets right across the inlet plane rather than only in the pilot region showed an almost complete absence of localised extinction in the Flame F results. As mentioned above, transport in the cross stream direction plays a key role in extinguishing CMC cells and if burning flamelets are injected into the primary jet rather than inert flamlets this mechanism is effectively switched off.

The present simulations highlight further the capabilities of the LESCMC approach to capture flames very close to extinction and suggest that further research is needed for modelling the scalar dissipation rate.

\section{Acknowledgements}

This work has been funded by the European Commission (Project "TECCAE").

\section{References}

Ayache, S.V., Mastorakos, E., 2012. Conditional moment closure/large eddy simulation of the Delft III natural gas non-premixed jet flame. Flow Turbulence and Combustion 88, $207-231$. 
Barlow, R.S., Frank, J., 1998. Effects of turbulence on species mass fractions in methane-air jet flames. Proceedings of the Combustion Institute 27, 1087-1095.

Branley, N., Jones, W.P., 2001. Large Eddy Simulation of a turbulent nonpremixed flame. Combustion and Flame 127, 1914-1934.

Brown, P.N., Hindmarsh, A.C., 1989. Reduced storage matrix methods in stiff ODE systems. Journal of Computational and Applied Mathematics 31, 40-91.

Cao, R.R., Pope, S.B., 2005. The influence of chemical mechanisms on PDF calculations of nonpremixed piloted jet flames. Combustion and Flame 143, 450-470.

Chung, T.J., 2002. Computational fluid dynamics. Cambridge University Press, Cambridge.

Cook, A.W., Riley, J.J., 1994. A subgrid model for equilibrium chemistry in turbulent flows. Physics of Fluids 6, 2868-2870.

Di Mare, F., Jones, W.P., Menzies, K.R., 2004. Large eddy simulation of a model gas turbine combustor. Combustion and Flame 137, 278-294.

Garmory, A., Mastorakos, E., 2011. Capturing localised extinction in sandia flame f with LES-CMC. Proceedings of the Combustion Institute 33, 1673 -1680 .

Germano, M., Piomelli, U., Moin, P., Cabot, W., 1991. A dynamic subgridscale eddy viscosity model. Physics of Fluids 3, 1760-1765. 
Ihme, M., Pitsch, H., 2008. Prediction of extinction and reignition in nonpremixed turbulent flames using a flamelet/progress variable model 2. Application in LES of Sandia flames D and E. Combustion and Flame 155, 90-107.

James, S., Zhu, J., Anand, M.S., 2006. Large-eddy simulations as a design tool for gas turbine combustion systems. AIAA Journal 44, 674-686.

Jiménez, C., Ducros, F., Cuenot, B., Bédat, B., 2001. Subgrid scale variance and dissipation of a scalar field in large eddy simulations. Physics of Fluids $13,1748-1754$.

Jones, W.P., Prasad, V.N., 2010. Large eddy simulation of the Sandia flame series (D-F) using the eulerian stochastic field method. Combustion and Flame 157, 1621-1636.

Karpetis, A.N., Barlow, R.S., 2005. Measurements of flame orientation and scalar dissipation in turbulent partially premixed methane flames. Proceedings of the Combustion Institute 30, 665-672.

Klimenko, A.Y., Bilger, R.W., 1999. Conditional Moment Closure for turbulent combustion. Progress in Energy and Combustion Science 25, 595-687.

Lindstedt, R.P., Louloudi, S.A., Váos, E.M.., 2000. Progress-variable approach for large-eddy simulation of non-premixed turbulent combustion. Proceedings of the Combustion Institute 28, 149-156.

Mustata, R., Valiño, L., Jiménez, C., Jones, W.P., Bondi, S., 2006. A Probability Density Function Eulerian Monte Carlo field method for Large-eddy 
simulations. Application to a turbulent piloted methane/air diffusion flame (Sandia D). Combustion and Flame 145, 88-104.

Navarro-Martinez, S., Kronenburg, A., 2007. LES-CMC simulations of a turbulent bluff-body flame. Proceedings of the Combustion Institute 31, 1721-1728.

Navarro-Martinez, S., Kronenburg, A., 2009. LES-CMC simulations of a lifted methane flame. Proceedings of the Combustion Institute 32, 1509 1516.

Navarro-Martinez, S., Kronenburg, A., Di Mare, F., 2005. Conditional Moment Closure for Large Eddy Simulations. Flow, Turbulence and Combustion $75,245-274$.

O’Brien, E., Jiang, T.L., 1991. The conditional dissipation rate of an initially binary scalar in homogeneous turbulence. Physics of Fluids 3, 3121-3123.

Pierce, C.D., Moin, P., 1998. A dynamic model for subgrid-scale variance and dissipation rate of a conserved scalar. Physics of Fluids 10, 3041-3044.

Pierce, C.D., Moin, P., 2004. Progress-variable approach for large-eddy simulation of non-premixed turbulent combustion. Journal of Fluid Mechanics $504,73-97$.

Pitsch, H., Steiner, H., 2000. Large-eddy simulation of a turbulent piloted methane/air diffusion flame (Sandia flame D). Physics of Fluids 12, 25412554 . 
Schneider, C., Dreizler, A., Janicka, J., Hassel, E., 2003. Flow field measurements of stable and locally extinguishing hydrocarbon-fuelled jet flames. Combustion and Flame 135, 185-190.

Sung, C.J., Law, C.K., Chen, J.Y., 1998. An augmented reduced mechanism for methane oxidation with comprehensive global parametric validation. Proceedings of the Combustion Institute 27, 295-304.

Triantafyllidis, A., Mastorakos, E., 2010. Implementation issues of the Conditional Moment Closure model in Large Eddy Simulations. Flow Turbulence and Combustion 84, 481-512.

Triantafyllidis, A., Mastorakos, E., Eggels, R., 2009. Large Eddy Simulations of forced ignition of a non-premixed bluff-body methane flame with Conditional Moment Closure. Combustion and Flame 156, 2328-2345.

Vogiatzaki, K., Kronenburg, A., Navarro-Martinez, S., Jones, W.P., 2011. Stochastic multiple mapping conditioning for a piloted, turbulent jet diffusion flame. Proceedings of the Combustion Institute 33, 1523-1531.

Xu, J., Pope, S.B., 2000. PDF calculations of turbulent nonpremixed flames with local extinction. Combustion and Flame 123, 281-307. 

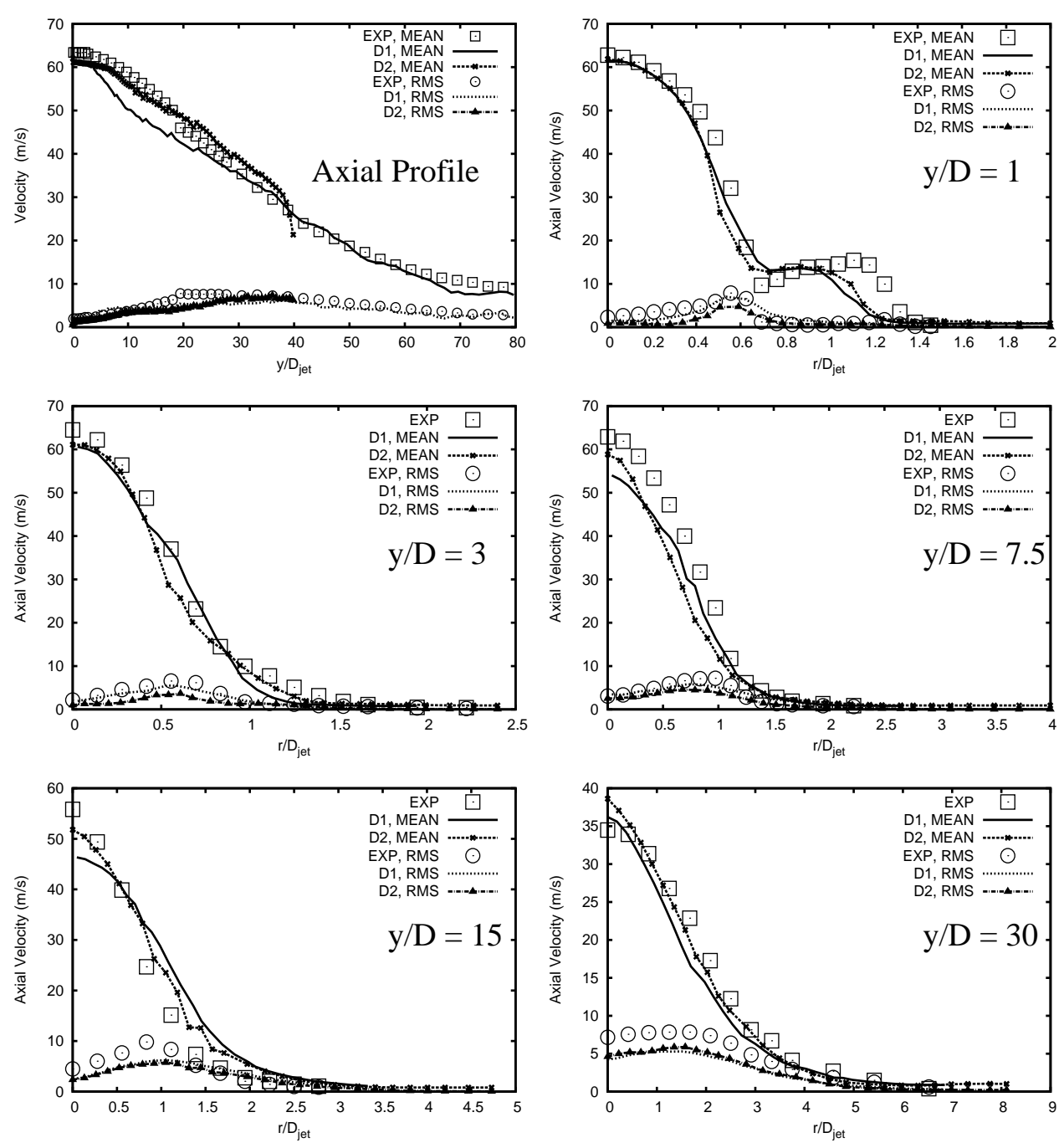

Figure 1: Axial and radial profiles of velocity for Flame D from experiment (Schneider et al., 2003) and LES-CMC solutions using coarse LES grid (D1) and fine grid (D2). 

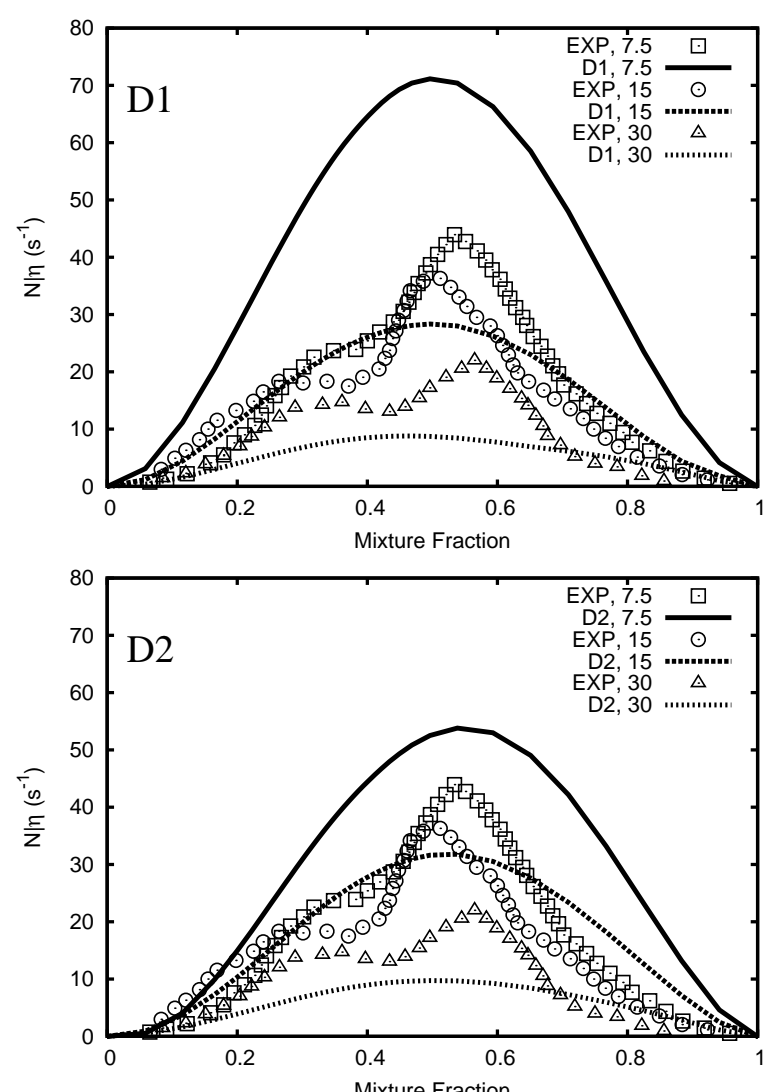

Figure 2: Conditional scalar dissipation rate for Flame D using coarse grid, D1, and fine grid, D2 Experimental data from Karpetis and Barlow (2005). 

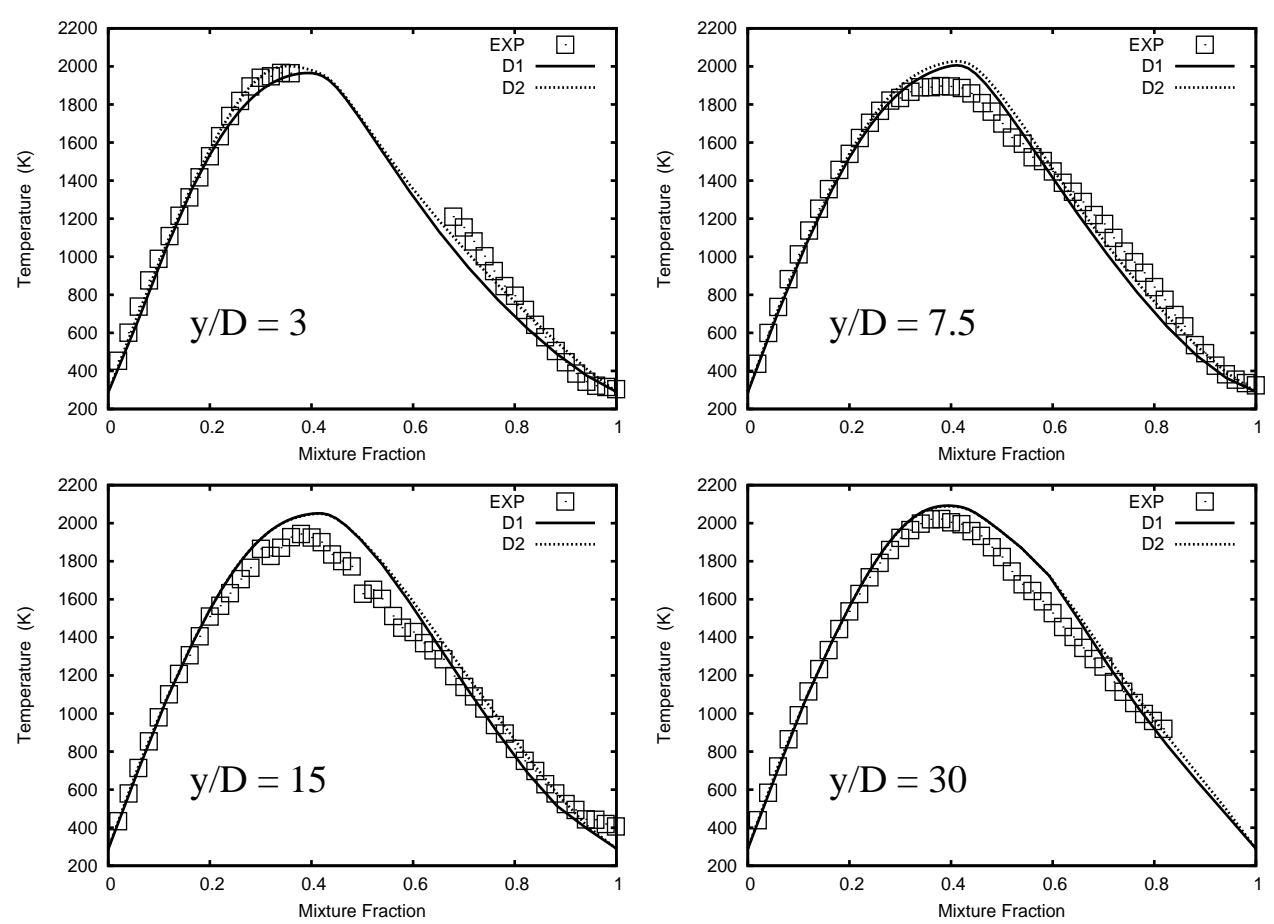

Figure 3: Conditional temperature for Flame D predicted by CMC using simulations D1 and D2. Experimental data from Barlow and Frank (1998). 

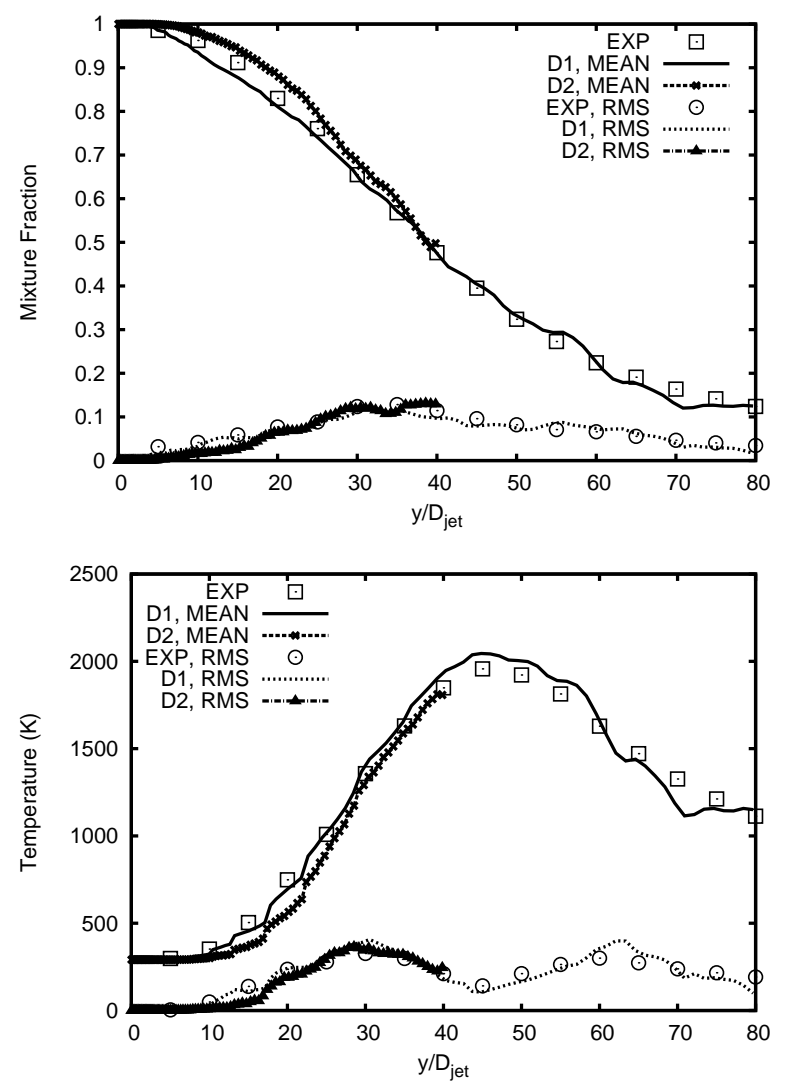

Figure 4: Mean mixture fraction and unconditional temperature along centre line. Experimental data from Karpetis and Barlow (2005). 

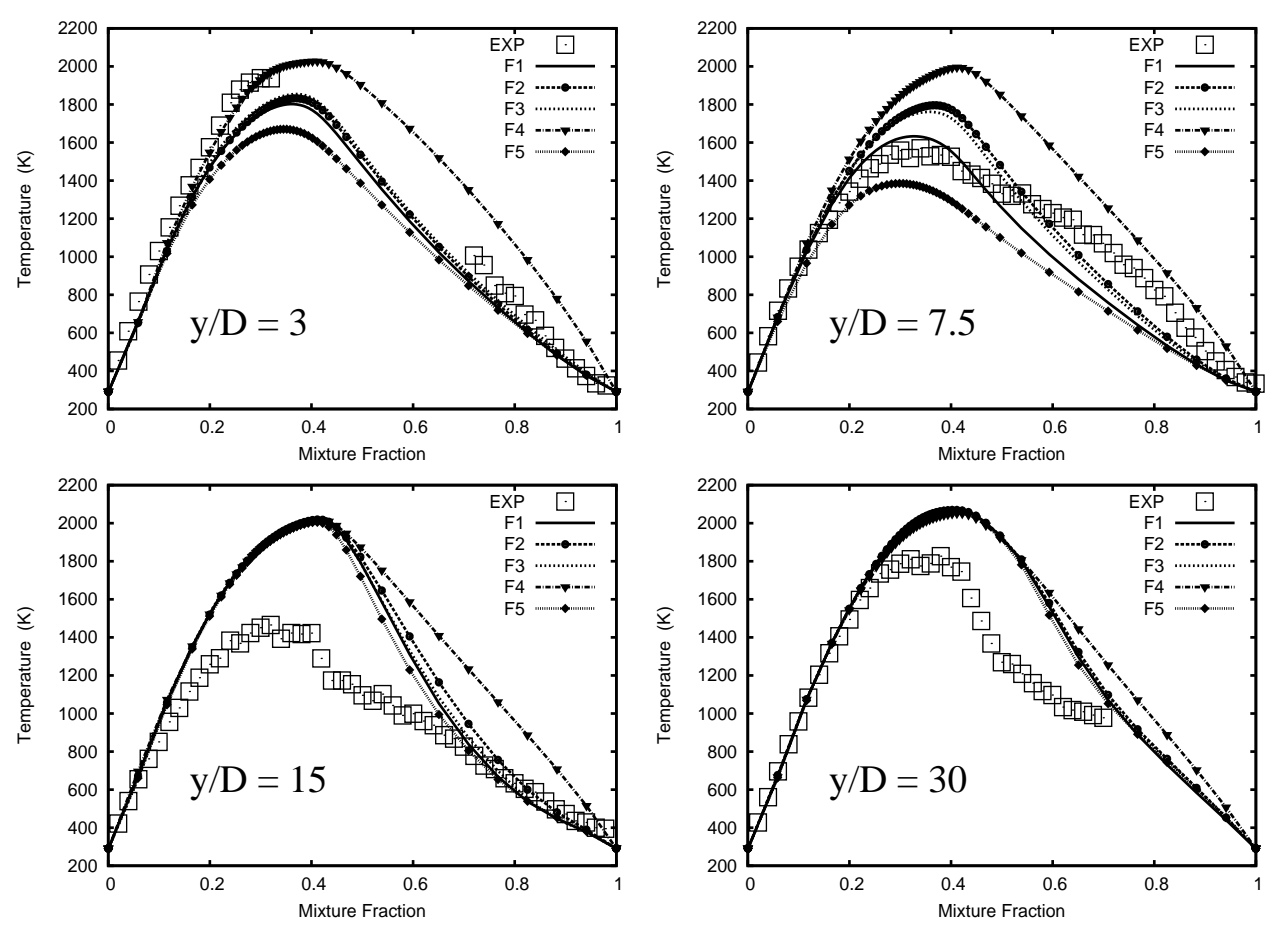

Figure 5: Conditional temperature for Flame F predicted by CMC. Experimental data from Barlow and Frank (1998). 

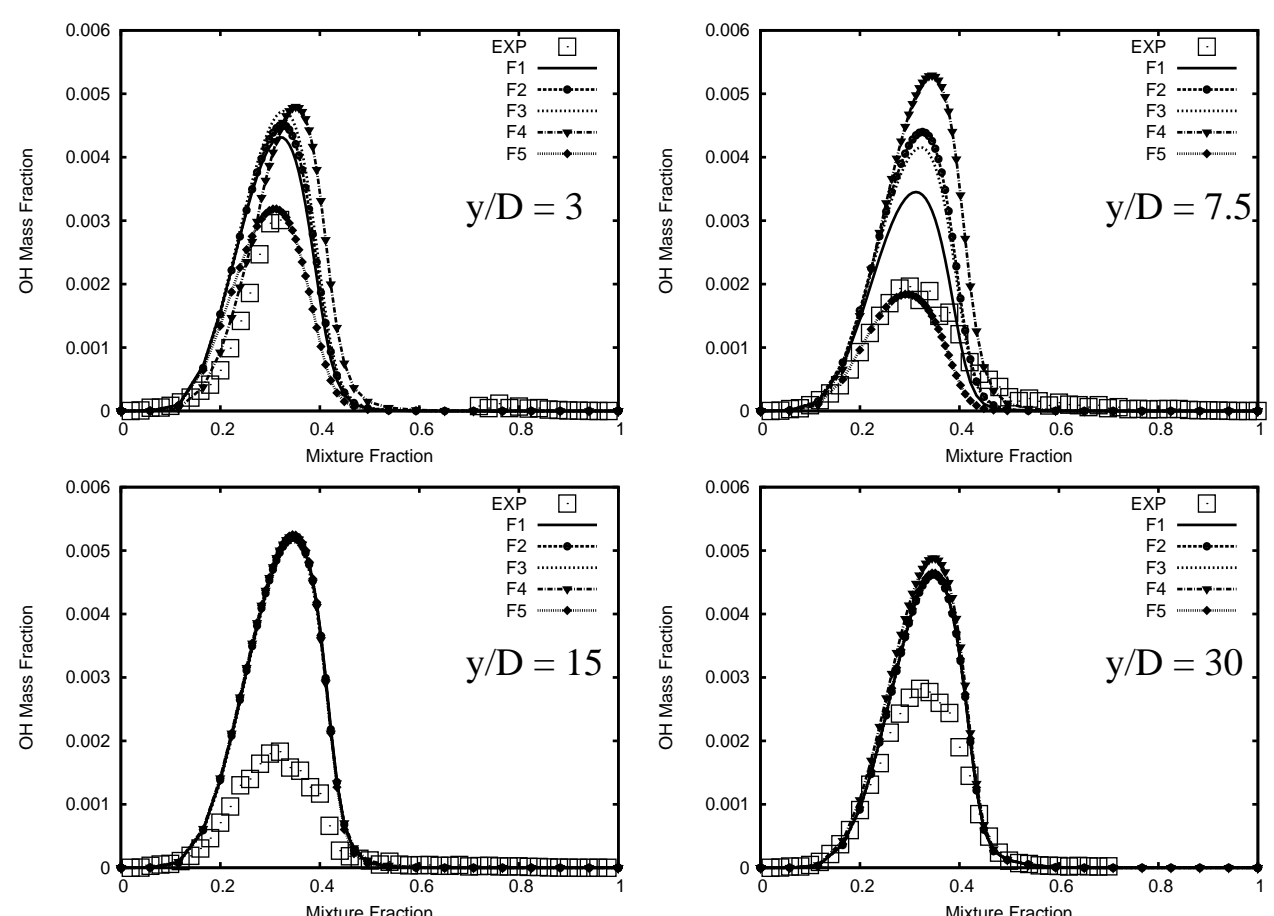

Figure 6: Conditional $O H$ for Flame F predicted by CMC. Experimental data from Barlow and Frank (1998). 


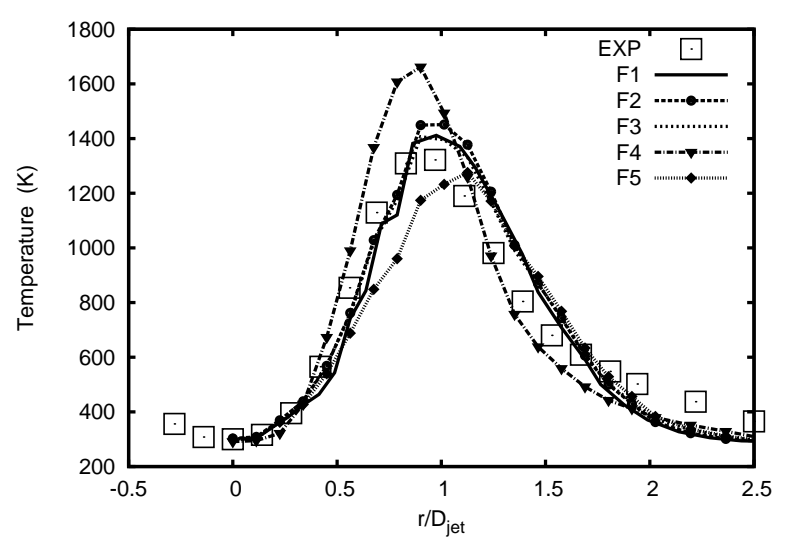

Figure 7: Radial profiles of mean unconditional temperature at $y / D=7.5$ for Flame F. Results are shown for LES-CMC calculations using the five options listed in Table 3 together with experimental data from Barlow and Frank (1998).

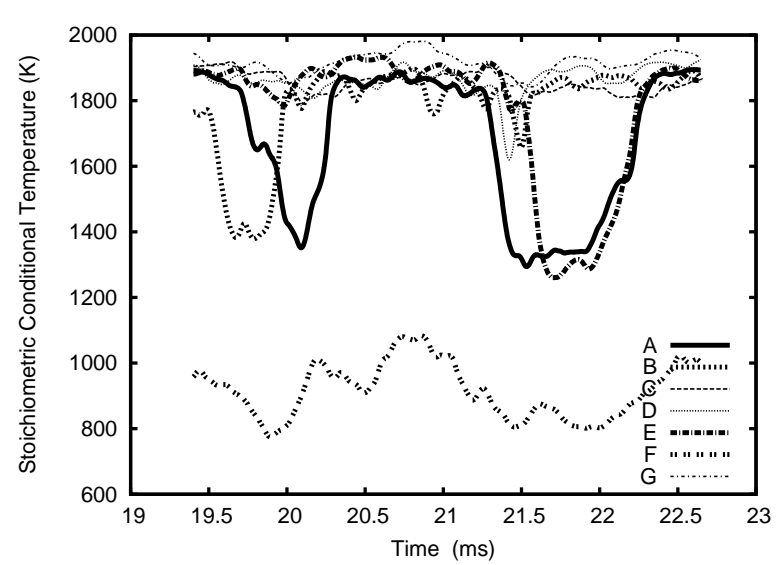

Figure 8: Time series of stoichiometric conditional temperature for several CMC cells. 'A' is located at $y / D=6.6$ and $r / D=0.9$, ' $\mathrm{B}$ ' and ' $\mathrm{C}$ ' are up and downstream respectively in the y direction, ' $\mathrm{D}$ ' and ' $\mathrm{E}$ ' neighbour ' $\mathrm{A}$ ' in the tangential direction and ' $\mathrm{F}$ ' and ' $\mathrm{G}$ ' are neighbours in the radial direction with ' $\mathrm{F}$ ' closer to the axis. 




Figure 9: Instantaneous isosurface, taken at $21.8 \mathrm{~ms}$, of stoichiometric mixture fraction coloured by LES resolved $O H$ mass fraction. Also marked are the locations of the seven CMC cells whose time series are shown in Fig. 8. 

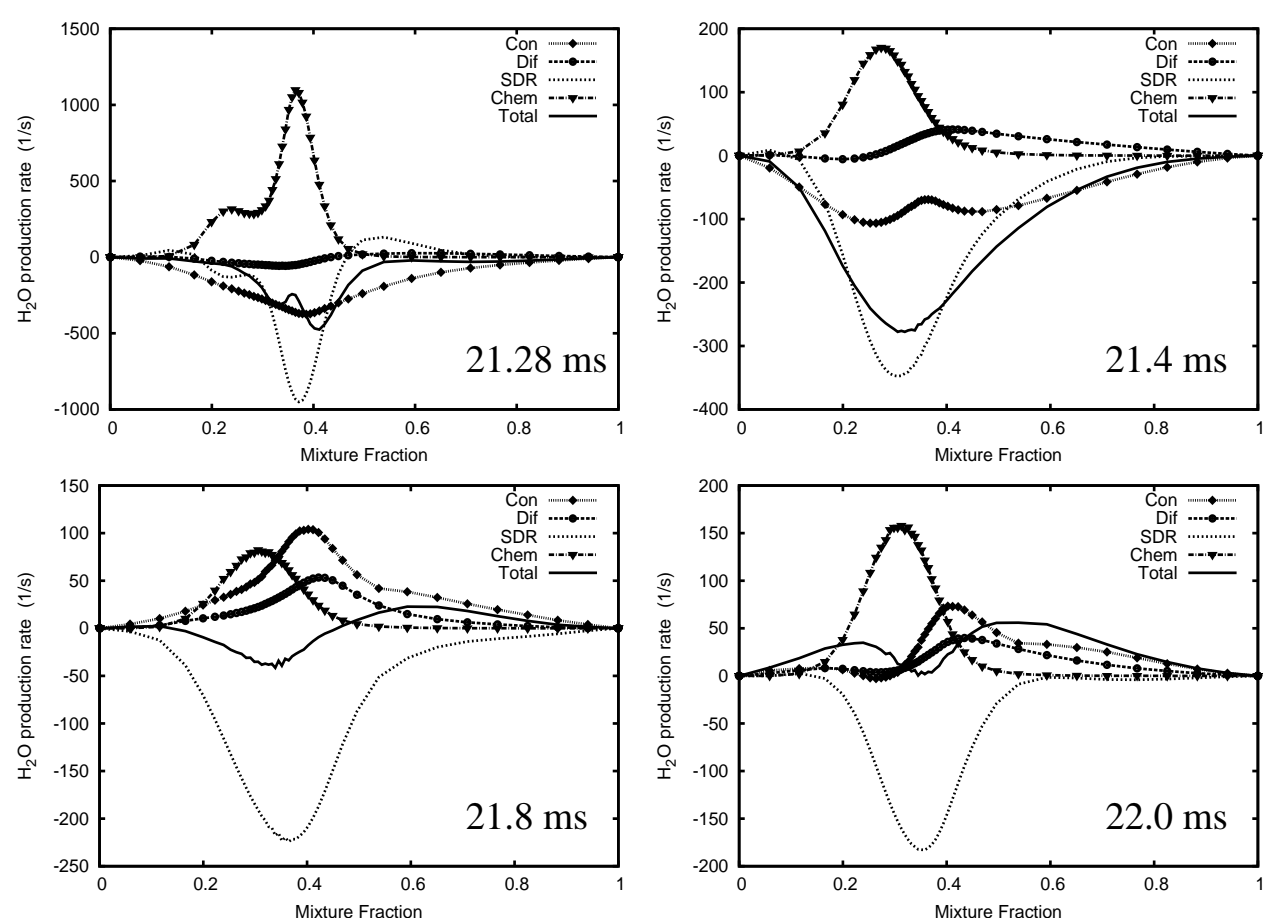

Figure 10: Contributions to conditional $\mathrm{H}_{2} \mathrm{O}$ production for Flame F predicted by CMC at node 'A' from Fig. 8. 'Con' is convection term, 'Dif' is diffusion is physical space, 'SDR' is the contribution due to conditional dissipation rate, 'Chem' is the rate of production by chemistry and 'Total' is the sum of these terms.

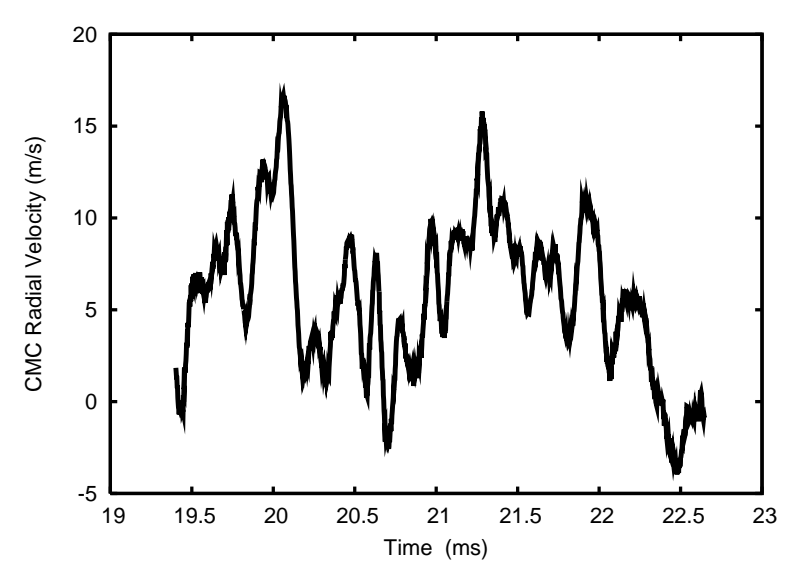

Figure 11: Time series of CMC radial velocity for node 'A.' 